\title{
Invarianza factorial y temporal del Cuestionario de Regulación Emocional (ERQ)
}

\author{
David Pineda ${ }^{1}$, Rosa M. Valiente ${ }^{1}$, Paloma Chorot ${ }^{1}$, José Antonio Piqueras ${ }^{2}$ y Bonifacio Sandín ${ }^{1}$ \\ ${ }^{1}$ Universidad Nacional de Educación a Distancia, Madrid, España \\ ${ }^{2}$ Universidad Miguel Hernández de Elche, Alicante, España
}

Factorial and temporal invariance of a Spanish version of the Emotional Regulation Questionnaire (ERQ)

\begin{abstract}
The Emotional Regulation Questionnaire (ERQ; Gross y John, 2003) is widely used to assess individual differences in reappraisal and suppression strategies of emotion regulation. The present study tests the factorial and temporal invariance of a Spanish adaptation of the ERQ on two samples of 1.980 participants aged 18-67 years. Psychometric properties, reliability, and factorial and temporal validity of the ERQ were calculated. Results of the confirmatory factor analysis showed the expected two-factor structure. Results also indicated adequate internal consistency, test-retest reliability, and convergent and discriminant validity. As expected, reappraisal use was positively associated with positive emotion and negatively with negative emotion, whereas suppression use was negatively associated with positive emotion and positively with negative emotion. These findings suggest that the Spanish version of the ERQ is a valid and reliable instrument for evaluating strategies of emotion regulation in the Spanish-speaking population.
\end{abstract}

Keywords: ERQ; emotion regulation; cognitive reevaluation; emotional suppression; assessment.

Resumen: El Cuestionario de Regulación Emocional (ERQ; Gross y John, 2003) es ampliamente utilizado para evaluar las diferencias individuales en las estrategias de regulación emocional reevaluación y supresión. El presente estudio examina la invarianza factorial y temporal de una adaptación española del ERQ en dos muestras de 1.980 participantes de 18-67 años de edad. Examinamos su fiabilidad, y validez factorial y temporal. El análisis factorial confirmatorio reflejó una estructura de dos factores. También encontramos niveles adecuados de consistencia interna, fiabilidad test-retest, y validez convergente y discriminante. Como se esperaba, el uso de la reevaluación se asoció positivamente con las emociones positivas y negativamente con las emociones negativas, mientras que la supresión se asoció a un patrón opuesto. Estos datos sugieren que la versión española del ERQ es un instrumento válido y fiable para evaluar las estrategias de regulación emocional en población de habla española.

Palabras clave: ERQ; regulación emocional; reevaluación cognitiva; supresión emocional; evaluación.

\section{Introducción}

Existe evidencia en la literatura que sugiere que las personas mantienen un cierto margen de control sobre las emociones, usando una amplia gama de estrategias para influir sobre cómo y cuándo se sienten estas emociones; de modo que el individuo es capaz de supervisar,

Recibido: 20 de octubre de 2017; aceptado: 25 de febrero de 2018 Correspondencia: Paloma Chorot, Universidad Nacional de Educación a Distancia, Facultad de Psicología, Juan del Rosal 10, 28040 Madrid. Correo-e: pchorot@psi.uned.es valorar y modificar los procesos involucrados en la génesis de la emoción y puede de este modo, modular sus manifestaciones. Se han constatado diferencias individuales en la capacidad para regular estas emociones, siendo algunos estrategias más exitosas que otros (Mayer, Caruso, y Salovey, 1999; Salovey y Mayer, 1990).

La regulación emocional fue conceptualizada por Gross (2002) como la capacidad que tiene el individuo para implementar estrategias que modifiquen sus experiencias emocionales, la expresión de sus propias emociones o su respuesta fisiológica para dar respuesta a las demandas ambientales. Dentro del abanico de estrate- 
gias de regulación emocional, la reevaluación cognitiva integra aquellas estrategias con las que el individuo es capaz de modificar la forma en que percibe, siente o es capaz de modificar, las emociones que experimenta ante una situación dada; este tipo de estrategias se centran en los antecedentes. Por otro lado, la supresión emocional se refiere a la capacidad de sujeto para inhibir o modular la respuesta cognitiva, conductual o fisiológica; en este caso se trataría de estrategias centradas en la respuesta (Gross y John, 2003).

La manera en que las personas regulan las emociones afecta a sus relaciones, su bienestar y percepción y experiencia del estrés (Gross, 2002; Lopes, Salovey, Côté, y Beers, 2005;), siendo la regulación emocional, junto a otros constructos transdiagnósticos primarios (Martínez-Vispo, Fernández del Río, López-Durán, y Becoña, 2016; Sánchez-Arribas, Chorot, Valiente, y Sandín, 2015), un concepto clave en el estudio de la psicopatología (Gil-Sanz et al., 2017; Jiménez, Orgambídez, y Pascual, 2015; Segura et al., 2017). Desde este marco, se han conceptualizado las estrategias de regulación emocional como adaptativas o desadaptativas en función de la relación que mantienen con los síntomas psicopatológicos (Aldao, 2012). Esta autora ha considerado como desadaptativas aquellas estrategias positivamente asociadas con los síntomas (p.ej., supresión, rumiación, preocupación, evitación) y adaptativas aquellas que correlacionan negativamente con los síntomas (p.ej., reevaluación, aceptación, resolución de problemas). A su vez, las distintas estrategias se han relacionado de manera diferencial con algunos trastornos. Así por ejemplo, la rumiación se ha relacionado con trastornos como los episodios depresivos, la ansiedad generalizada, el trastorno de estrés postraumático o la ansiedad social (McLaughlin y Nolen-Hoeksema, 2011).

La relación etiopatogénica que mantienen las estrategias de regulación emocional con distintos trastornos ha llevado a conceptualizarlas como procesos transdiagnósticos (Aldao, 2012; Aldao y Nolen-Hoeksema, 2010; Aldao, Nolen-hoeksema, y Schweizer, 2010; Sandín, Chorot, y Valiente, 2012), e incluirlas en los nuevos protocolos de terapia cognitivo-conductual transdiagnóstica (p.ej., García-Escalera et al., 2017; Grill, Castañeiras, y Fasciglione, 2017; Rosique, Sandín, Valiente, García-Escalera, y Chorot, en prensa). Tratamiento transdiagnóstico de un caso clínico con elevada comorbilidad de trastornos emocionales: Aplicación del Protocolo Unificado. Revista Argentina de Clínica Psicológica). Diversos autores han puesto de manifiesto la estrecha relación que mantiene la regulación emocional con los trastornos de ansiedad y del estado de ánimo (Amstadter, 2008; Martin, Dahlen, Martin, y Dahlen, 2005). Desde esta perspectiva transdiagnós- tica, la regulación emocional resulta un concepto clave en la explicación de la alta comorbilidad presente en los trastornos emocionales (Cummings, Caporino, y Kendall, 2014; Piqueras, Martín-Vivar, Sandín, San Luis, y Pineda, 2017; Sandín et al., 2012). Por ello, la evaluación de la regulación emocional constituye un tema de especial interés en el momento actual.

Gross y John (2003) desarrollaron el Emotion Regulation Questionaire (ERQ) como una prueba de autoinforme para la evaluación de dos dimensiones básicas de la regulación emocional. El cuestionario ha sido traducido a 26 idiomas, incluidos el chino, el alemán, el portugués, el italiano, el ruso y el español, entre otros (Stanford Psychophysiology Laboratory, 2010). Muchas de estas traducciones cuentan con trabajos de validación. Una primera traducción de la versión española fue llevada a cabo por Rodríguez-Carvajal, Moreno-Jiménez y Garrosa (2006). Posteriormente, Cabello, Salguero, Fernández-Berrocal y Gross (2013) publicaron datos de validación del cuestionario basados en una traducción diferente del mismo. En este trabajo los autores obtuvieron una estructura factorial de dos factores independientes, confirmado la estructura de la escala original (Gross y John, 2003). La fiabilidad de las subescalas fue también adecuada, con un alfa de Cronbach de .75 para supresión emocional y .79 para la reevaluación cognitiva. La validez convergente se valoró con otras medidas de regulación emocional, mientras que la validez divergente se examinó explorando las relaciones con los rasgos de personalidad según el modelo de los cinco grandes (Cabello et al., 2013). La importancia de probar la hipótesis de la invariancia se ha argumentado ampliamente (Cheung y Rensvold, 2002; Meade y Lautenschlager, 2004; Pineda, Martín-Vivar, Sandín, y Piqueras, 2018). Otro supuesto psicométrico importante y necesario para interpretar los cambios en las puntuaciones en distintas aplicaciones de un test, como cambios en la puntuación del constructo que miden, es que sea evaluada de la misma manera en múltiples ocasiones, un requisito conocido como invarianza temporal o invarianza de medida longitudinal (Meredith, 1993; Widaman, Ferrer, y Conger, 2010). La invarianza factorial de la versión original ha sido probada con muestras de diferentes grupos étnicos, mostrando ser invariante para los grupos y en función del sexo (Melka, Lancaster, Bryant, y Rodriguez, 2011). Sin embargo, la invarianza factorial de la versión española del ERQ aún no ha sido comprobada. Por otro lado, también es necesario el estudio de la invariancia temporal para determinar si los ítems del ERQ evalúan el mismo atributo a lo largo del tiempo.

Se trata por tanto de una escala de indudable interés para la psicología clínica en nuestro país, que justifica el 
estudio de sus propiedades psicométricas. Que nosotros sepamos, hasta estos momentos sólo el trabajo de Cabello et al. (2013) ha abordado este tema proporcionando datos sobre algunas de las propiedades psicométricas del cuestionario. No obstante, se precisan nuevos estudios que examinen tanto su invarianza factorial, como la consistencia temporal de su estructura factorial, así como también proporcionar datos normativos referidos a la baremación de las puntuaciones en el cuestionario.

El objetivo general de este trabajo fue proporcionar evidencia consistente sobre la validez de la versión española del ERQ. Los objetivos específicos fueron: (a) examinar la estructura factorial de la escala, (b) aportar nuevos datos de su validez referidos a medidas de síntomas psicopatológicos, y (c) proporcionar datos normativos aplicables a la población española. Puesto que se trata de una prueba que cuenta con trabajos que ponen de manifiesto sus propiedades psicométricas tanto para la prueba original como para una de las versiones traducidas, como hipótesis de partida nos planteamos que la versión española del ERQ: (1) presentará una estructura de dos factores no relacionados; (2) la proporción de varianza media explicada por los factores de la ERQ será mayor de .50 (validez convergente y discriminante); (3) la subescala de supresión emocional tendrá una asociación positiva con diversos síntomas de trastornos psiquiátricos, mientras que la subescala de reevaluación cognitiva mostrará una asociación negativa con los síntomas de trastornos; (4) deberíamos obtener índices de fiabilidad adecuados, similares a los obtenidos en anteriores validaciones; y (5) finalmente la ERQ presentará valores de ajuste adecuados de invarianza factorial temporal y en función del sexo.

\section{Método}

\section{Participantes}

Para el presente trabajo se utilizaron dos muestras independientes de estudiantes universitarios de los dos primeros cursos de psicología de la Universidad Nacional de Educación a Distancia, que participaron de forma voluntaria en la investigación. La primera muestra estuvo compuesta por 997 participantes de los cuales 780 eran mujeres $(78.23 \%)$. La edad de los participantes estuvo comprendida entre los 19 y los 67 años $(M=32.41$; $D T$ =9.56). La segunda muestra estuvo formada por $983 \mathrm{su}-$ jetos de los cuales 768 fueron mujeres (78.12\%). La edad de los participantes estuvo comprendida entre los 18 y los 61 años $(M=33.14 ; D T=9.99)$. Los estadísticos descriptivos de las diferentes submuestras se indican en la Tabla 1. La distribución de participantes por regiones de España se muestra en la Tabla 2.
Tabla 1. Estadísticos descriptivos de las muestras

\begin{tabular}{lcc}
\hline & Muestra 1 & Muestra 2 \\
\hline$n$ & 997 & 983 \\
Mujeres (\%) & $780(78.2)$ & $768(78.1)$ \\
Edad, $M(D T)$ & $32.41(9.56)$ & $33.14(9.98)$ \\
Rango de edad & $19-67$ & $18-61$ \\
Nivel socioeconómico, $n(\%):$ & & \\
$\quad$ Bajo & $268(27.0)$ & $256(26.0)$ \\
$\quad$ Medio & $623(62.5)$ & $619(63.0)$ \\
$\quad$ Alto & $106(11.5)$ & $108(11.0)$ \\
Estado civil, $n(\%):$ & & \\
$\quad$ Soltero & $550(55.2)$ & $556(56.6)$ \\
$\quad$ En pareja & $379(38.0)$ & $356(36.2)$ \\
$\quad$ Separado/divorciado/viudo & $68(6.8)$ & $71(7.2)$ \\
\hline
\end{tabular}

Tabla 2. Distribución de participantes según regiones ( $n$ y \%)

\begin{tabular}{lcc}
\hline \multicolumn{1}{c}{ Provincia } & $\begin{array}{c}\text { Muestra } 1 \\
(n=997)\end{array}$ & $\begin{array}{c}\text { Muestra } 2 \\
(n=983)\end{array}$ \\
\hline Andalucía & $159(15.95 \%)$ & $135(13.73 \%)$ \\
Aragón & $83(8.32 \%)$ & $61(6.21 \%)$ \\
Asturias & $35(3.51 \%)$ & $31(3.15 \%)$ \\
Baleares & $21(2.11 \%)$ & $25(2.54 \%)$ \\
Canarias & $47(4.71 \%)$ & $47(4.78 \%)$ \\
Cantabria & $18(1.81 \%)$ & $16(1.63 \%)$ \\
Castilla-León & $74(7.42 \%)$ & $52(5.29 \%)$ \\
Castilla-La Mancha & $53(5.32 \%)$ & $93(9.46 \%)$ \\
Cataluña & $43(4.31 \%)$ & $48(4.88 \%)$ \\
Extremadura & $16(1.60 \%)$ & $33(3.36 \%)$ \\
Galicia & $60(6.02 \%)$ & $65(6.61 \%)$ \\
La Rioja & $12(1.20 \%)$ & $8(0.81 \%)$ \\
Madrid & $228(22.87 \%)$ & $231(23.50 \%)$ \\
Murcia & $29(2.91 \%)$ & $26(2.64 \%)$ \\
Navarra & $16(1.60 \%)$ & $17(1.73 \%)$ \\
País Vasco & $29(2.91 \%)$ & $20(2.03 \%)$ \\
Valencia & $61(6.12 \%)$ & $74(7.53 \%)$ \\
Ceuta & $6(0.60 \%)$ & $1(0.10 \%)$ \\
Melilla & $7(0.70 \%)$ & $0(0.00 \%)$ \\
\hline & &
\end{tabular}

\section{Instrumentos}

Cuestionario de Regulación Emocional (Emotional Regulation Questionnaire, ERQ; Gross y John, 2003). 
Este cuestionario consta de 10 ítems en los que el sujeto debe expresar su grado de acuerdo en referencia a como suele regular sus emociones. El participante contesta según una escala Likert de siete puntos, que varía entre 1 («completamente en desacuerdo») y 7 («completamente de acuerdo»). La versión original del cuestionario presenta una estructura factorial de dos factores no relacionados. El primer factor recoge las puntuaciones de seis ítems, que evalúan la reevaluación cognitiva. El segundo de los factores hace referencia a la supresión emocional en incluye los cuatros ítems restantes que componen el cuestionario. Aplicamos la versión traducida al español por Sandín, Chorot, Valiente y Pineda (2012), con retrotraducción efectuada por un psicólogo clínico con lengua nativa inglesa. Los autores de la escala original obtuvieron unos índices de fiabilidad alfa de Cronbach de .79 para la escala de reevaluación cognitiva y .73 para la de supresión.

Cuestionario de Evaluación de Síntomas-45 (SA45; Davison et al., 1997; Sandín, Valiente, Chorot, Santed, y Lostao, 2008) El cuestionario consta de 45 ítems sobre síntomas psicopatológicos en los que el sujeto debe indicar hasta qué punto ha experimentado cada uno de los síntomas durante la última semana, según una escala Likert de cinco puntos que varía entre 0 («nada en absoluto») y 4 («mucho o extremadamente»). El cuestionario tiene las nueve subescalas siguientes: somatización, obsesión-compulsión, sensibilidad interpersonal, depresión, ansiedad, hostilidad, ansiedad fóbica (agorafobia), ideación paranoide, y psicoticismo. Se utilizó la versión española validada por Sandín, et al. (2008) . Los índices de fiabilidad con la Muestra 2 han resultado similares a los informados por los autores ( $\alpha$ de Cronbach; somatización $=.81$; obsesión-compulsión $=.78$; sensibilidad interpersonal $=.85$; depresión $=.89 ;$ ansiedad $=$ .83 ; hostilidad $=.76$; ansiedad fóbica $=.84$; ideación paranoide $=.78$; psicoticismo $=.62$ ) .

Escala de Tolerancia al Estrés (DTS; Simons y Gaher, 2005; Sandín, Simons, Valiente, Simons, y Chorot, 2017 ). La DTS es una prueba de autoinforme de 15 ítems diseñada para evaluar el grado en que las personas experimentan y soportan estados psicológicos de estrés. Los participantes puntúan cada ítem en una escala Likert de cinco puntos que van desde «muy de acuerdo» (1) a «muy en desacuerdo» (5), donde las puntuaciones más bajas indican mayor capacidad para tolerar el malestar. Además de una puntuación total, la DTS incluye cuatro subescalas (tolerancia, evaluación, absorción y regulación). La versión española del cuestionario (Sandín et al., 2017) ha demostrado poseer adecuadas propiedades psicométricas ( $\alpha$ de Cronbach; tolerancia $=.83$; absorción $=.89$; evaluación $=.84$; regulación $=.83$ ). Los re- sultados del alfa de Cronbach obtenidos con la Muestra 2 han sido también adecuados (tolerancia $=.83$; absorción $=.86$; evaluación $=.84$; regulación $=.79$ ).

Escala de Alexitimia de Toronto (TAS-20; Bagby, Parker, y Taylor, 1994; Sandín, Chorot, Santed, y Jiménez 1996). Consiste en una escala de autoinforme de 20 ítems a los que el participante según una escala Likert de 5 puntos de 1 («nada de acuerdo») a 5 («totalmente de acuerdo»). Se compone de tres subescalas: (1) dificultad para identificar sentimientos y diferenciarlos de las sensaciones corporales o fisiológicas que acompañan la activación emocional; (2) dificultad para describir los sentimientos a los demás; y (3) un estilo de pensamiento orientado hacia lo externo. La adaptación española (Sandín et al., 1996) obtuvo una adecuada fiabilidad $(\alpha=.81)$ y validez. La fiabilidad del TAS-20 con la Muestra 2 ha resultado satisfactoria $(\alpha=.79)$

Escalas de PANAS de Afecto Positivo y Afecto Negativo (PANAS; Sandín et al., 1999). Consta de 20 ítems, 10 referidos al afecto positivo y 10 al afecto negativo. Cada ítem se puntúa según una escala Likert de 1 («nada o casi nada») a 5 («muchísimo»). La escala de afecto positivo (AP) evalúa la capacidad de involucrarse en actividades placenteras. La escala de afecto negativo (AN) evalúa la presencia de estrés percibido. Se aplicó la versión española validada por Sandín et al. (1999). La prueba ha mostrado una adecuada fiabilidad con la Muestra 2 $(\mathrm{AP}=.91 ; \mathrm{AN}=.89)$.

\section{Procedimiento}

El reclutamiento de los participantes se llevó a cabo por muestreo incidental. Los sujetos recibieron créditos por su participación en la investigación, completando los cuestionarios a través de la plataforma online del grupo de investigación. La Muestra $1(n=997)$ cumplimentó el protocolo en un Tiempo 1 y, 6 meses más tarde, en un Tiempo $2(n=725)$. La Muestra $2(n=983)$ aplicó el protocolo en una sola ocasión. Los protocolos se aplicaron durante 2012. El estudio fue aprobado por el comité de bioética de la Universidad Nacional de Educación a Distancia.

\section{Análisis estadísticos}

Se realizaron comprobaciones sobre la adecuación de los datos para el análisis factorial. Para poner a prueba la validez del constructo, se realizó una serie de análisis factoriales confirmatorios (CFA). Estos análisis se basaron en la matriz de correlaciones de Pearson; se optó por aplicar el método de estimación de máxima verosimilitud robusto (MLR). Estudios de simulación han puesto de manifiesto que este método suele ser el más adecuado 
para escalas de cinco o más valores ordinales (Rhemtu1la, Brosseau-Liard, y Savalei, 2012).

Para establecer la validez convergente se calculó la varianza media extraída por los factores (AVE), valores mayores o iguales a $.50 \mathrm{se}$ consideran indicativos de validez (Fornell y Larcker, 1981). La validez discriminante se evaluó teniendo en cuenta que el AVE de cada factor debe ser mayor que la correlación de sí mismo con cualquier otro factor (Fornell y Larcker, 1981). Más evidencias de validez convergente y discriminante se obtuvieron tras el estudio de los patrones de correlaciones entre los factores de la escala y otros constructos próximos o lejanos de la misma.

Por último, para estimar la fiabilidad de la escala se examinó la consistencia interna y la estabilidad temporal de la prueba a los 6 meses. La fiabilidad de cada elemento se evaluó mediante el análisis de las correlaciones entre cada ítem y su respectivo factor (Hair, Sarstedt, Hopkins, y Kuppelwieser, 2014). Para determinar la consistencia interna, se realizaron análisis de la fiabilidad de ambas subescalas, basado en la matriz de correlaciones de Pearson; se calculó mediante el coeficiente alfa de Cronbach y el coeficiente omega de McDonald (este último se considera un estimador mayor de la fiabilidad que el alfa de Cronbach; Dunn, Baguley, y Brunsden, 2014). Para determinar la estabilidad temporal se analizó la invarianza temporal de la escala siguiendo el método de la invarianza progresiva (Dimitrov, 2010, 2012). Como índices de ajuste de la invarianza temporal se tuvo en cuenta la diferencia en el índice de ajuste comparativo (CFI), más robusto con muestras amplias que la diferencia en la Chi cuadrado (Cheung y Rensvold, 2002; Meade, Johnson, y Braddy, 2006, 2008).

Los análisis estadísticos se realizaron utilizando el programa R (R Core Team, 2016) y las librerías MVN (Korkmaz, Goksuluk, y Zararsiz, 2014) y Lavaan (Rosseel, 2012).

\section{Resultados}

Los resultados del análisis de normalidad reflejaron la presencia de cierta asimetría (rango $=-1.20,1.30)$ y curtosis (rango $=-1.40,1.20$ ), estadísticamente significativa ( $\mathrm{SW}>.79 ; p<.001)$, si bien estas puntuaciones no se desvían en exceso de la curva normal. Los resultados del análisis de la adecuación muestral de los datos para el análisis factorial indican que el test de Bartlett para el contraste de la esfericidad nos permite rechazar la hipótesis de que la matriz de correlación poblacional es identidad (Bartlett $\left.{ }_{(\mathrm{gl}=9)}=705.22 ; p>.001\right)$. El valor del test Kaiser-Meyer-Olkin $(\mathrm{KMO}=.80)$ sugiere que la matriz de correlaciones es apropiada para continuar con el análisis factorial.

\section{Análisis factorial confirmatorio del ERQ}

En un primer paso se analizaron los modelos de dos factores correlacionados y no correlacionados propuestos por Gross y Thomson (2007) en su trabajo de validación inicial. Como puede verse en la Tabla 3, apenas se aprecian diferencias en cuanto a los índices de ajuste de ambos modelos. Para tratar de mejorar el ajuste del modelo y, tras comprobar los índices de modificación, se realizaron una serie de análisis confirmatorios de modelos alternativos. En un primer paso se correlacionaron los errores de los ítems 4 y 5. Esta correlación está justificada puesto que ambos ítems saturan en la misma escala, además ambos expresan la capacidad del sujeto para cambiar el pensamiento con la intención de modificar sus emociones. Los valores de ajuste del modelo, aun habiendo mejorado considerablemente, siguen sin ser óptimos RMSEA >.5, $\chi^{2} / \mathrm{gl}>4$ ). En un segundo paso se correlacionaron los errores de los ítems 3 y 4 . Esta correlación también estaría justificada dado que ambos ítems están expresados en positivo y ambos saturan en la escala de RC. Finalmente, el modelo de dos factores no correlacionados con dos errores correlacionados es el que presenta los mejores índices de ajuste (véase la Tabla 3).

\section{Validez convergente y discriminante}

Para comprobar la evidencia de validez convergente se calculó el AVE de los factores. El AVE para el factor Reevaluación cognitiva (AVE $=.47)$ no alcanzó el valor de .50; sin embargo, la diferencia encontrada entre el valor y el criterio ha resultado ser mínima. El valor del AVE para el factor Supresión emocional ha sido satisfactorio $(\mathrm{AVE}=.72)$. Los valores del AVE de los factores comparados con las correlaciones entre los factores han resultado adecuados (véase la Tabla 4), siendo la correlación entre los factores prácticamente nula $(r=-.04)$.

Con el objetivo de obtener información adicional sobre la validez convergente y discriminante, se calcularon las correlaciones de Pearson entre las dos subescalas del ERQ y las puntuaciones en el resto de variables incluidas en el estudio (véase la Tabla 5). La escala de reevaluación cognitiva del ERQ se ha relacionado positiva y significativamente con la escala de evaluación del DTS $(r=.24)$. También se ha encontrado que la reevaluación está relacionada negativa y significativamente con los síntomas psicopatológicos en general $(r=-.23)$ y en particular con aquellos en los que la evaluación de la situación o el control de impulsos juega un papel importante en su desarrollo como son la hostilidad $(r=-.24)$, la sensibilidad interpersonal $(r=-.23)$, la ansiedad $(r=-.20)$ o la agorafobia $(r=-.20)$. En esta misma línea, los resultados han mostrado una relación positiva y estadística- 
Tabla 3. Índices de bondad de ajuste para los diferentes modelos relacionados con la estructura factorial de la ERQ, basados en los análisis factoriales confirmatorios $(n=997)$

\begin{tabular}{|c|c|c|c|c|c|c|c|c|}
\hline $\begin{array}{l}\text { Modelo de análisis factorial } \\
\text { confirmatorio de la ERQ }\end{array}$ & $\begin{array}{l}\text { S-B } \chi^{2} \\
(\mathrm{gl})\end{array}$ & $\chi^{2} / \mathrm{gl}$ & RMSEA & SRMR & CFI & GFI & NFI & AIC \\
\hline $\begin{array}{l}\text { Modelo 1: Dos factores } \\
\text { correlacionados }\end{array}$ & $\begin{array}{c}733.23 \\
(34)\end{array}$ & 21.57 & .121 & .061 & .909 & .987 & .903 & 32501.58 \\
\hline $\begin{array}{l}\text { Modelo 2: Dos factores no } \\
\text { correlacionados }\end{array}$ & $\begin{array}{l}530.82 \\
(35)\end{array}$ & 15.16 & .119 & .063 & .909 & .987 & .903 & 32501.96 \\
\hline $\begin{array}{l}\text { Modelo 3: Dos factores no } \\
\text { correlacionados, errores 4-5 } \\
\text { correlacionados }\end{array}$ & $\begin{array}{c}220.17 \\
(34)\end{array}$ & 6.48 & .074 & .046 & .966 & .993 & .960 & 32193.31 \\
\hline $\begin{array}{l}\text { Modelo 4: Dos factores no } \\
\text { correlacionados, errores } 4-5 \text { y } 3-4 \\
\text { correlacionados }\end{array}$ & $\begin{array}{c}98.08 \\
(33)\end{array}$ & 2.97 & .044 & .042 & .988 & .997 & .982 & 32073.22 \\
\hline
\end{tabular}

Nota: Índices corregidos (método robusto). S-B $\chi^{2}=\chi^{2}$ escalado de Satorra-Bentler; RMSEA = root mean square error residual; SRMR $=$ standardized root mean square residual; $\mathrm{CFI}=$ comparative fit index; GFI = Goodness of fit index; NFI = Normed fit index; AIC = Akaike's information criterion.

mente significativa entre la reevaluación y el afecto positivo $(r=.28)$, y en sentido opuesto y con la misma magnitud con el afecto negativo $(r=-.28)$ y la alexitimia $(r=-.23)$. (véase la Tabla 5). La escala de supresión emocional mostró estar fuertemente relacionada con la alexitima $(r=.44)$ y en menor medida con el afecto positivo $(r=-.23)$. En lo relativo a las relaciones con la psicopatología, la supresión emocional ha mostrado una mayor asociación con la sintomatología interiorizada como la depresión ( $r=.22)$ o la sensibilidad interpersonal $(r=.24)$ (véase la Tabla 5).

\section{Fiabilidad}

Los resultados del análisis de fiabilidad de los ítems han mostrado una elevada correlación con su factor $(r>$ .70) a excepción del ítem 6 que ha presentado valores ligeramente inferiores $(r=.57)$ y prácticamente nulas o muy bajas con el otro factor de la escala $(r<-.12)$ (véase la Tabla 4).

Los resultados del análisis de la fiabilidad del cuestionario, tal como se derivan del análisis de las medidas de consistencia interna con el coeficiente alfa de Cronbach de ambas subescalas muestran unos resultados excelentes $(\alpha>$.84). Los resultados del cálculo de los coeficientes omega de McDonald también resultaron adecuados $(\omega>.77)$. La escala de supresión muestra una mayor consistencia a pesar de contar con dos ítems menos (véase la Tabla 4). Atendiendo a las cargas factoriales de los ítems con sus respectivos constructos vemos como el ítem 6 es que ha presentado la menor carga con .42 quedado el resto de valores entre el $.46 \mathrm{y}$ el .95 (véase Figura 1).

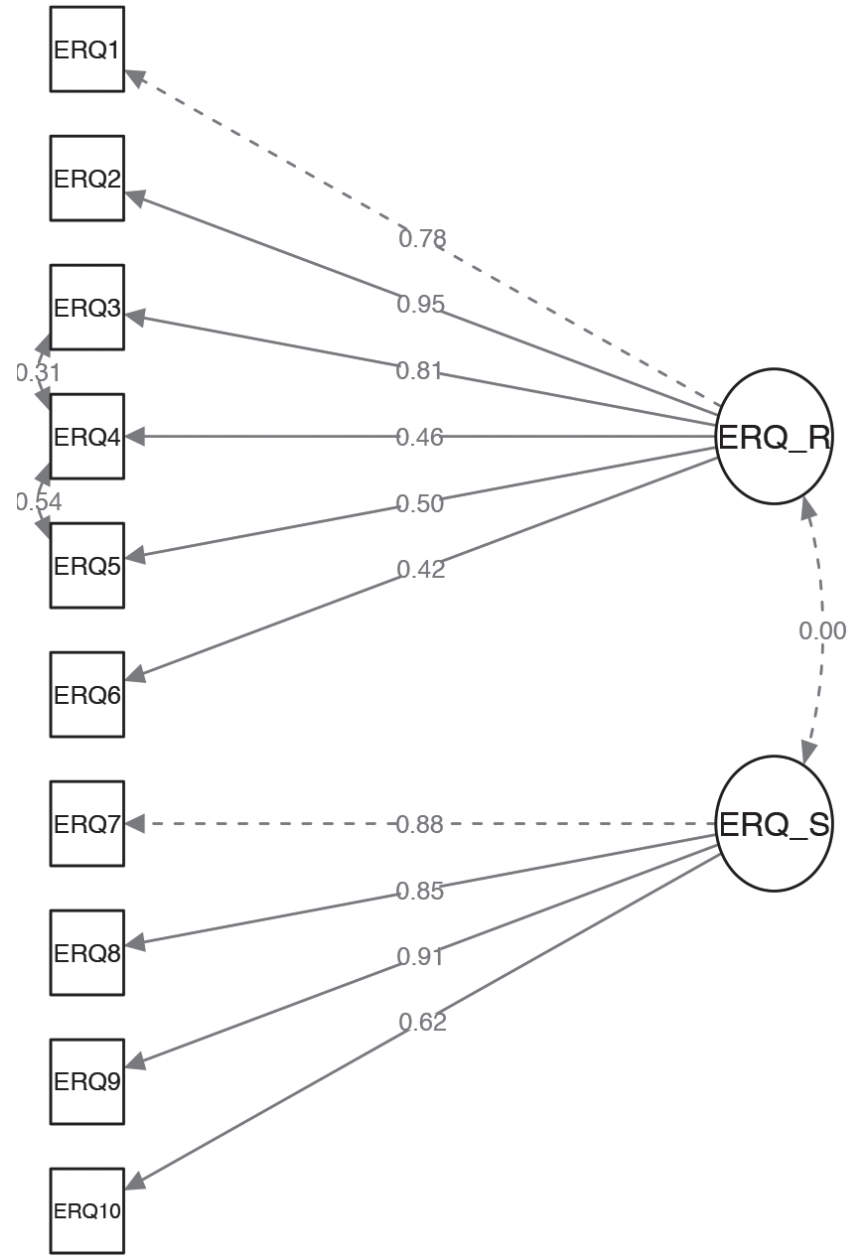

Figura 1. Estructura factorial y cargas factoriales estandarizadas de la ERQ. 
Tabla 4. Correlación de Pearson entre los ítems y los factores y varianza media extraída por los factores

\begin{tabular}{lccc}
\hline & $\begin{array}{c}\text { Reevaluación } \\
\text { cognitiva }\end{array}$ & $\begin{array}{c}\text { Supresión } \\
\text { emocional }\end{array}$ & $M(D T)$ \\
\hline Reevaluación cognitiva & $.47^{\mathrm{a}}$ & -.04 & $5.02(1.10)$ \\
$\begin{array}{l}\text { 1. Controlo mis emociones cambiando la forma de pensar acerca de } \\
\text { la situación en que estoy }\end{array}$ & $.77^{* * *}$ & -.01 & $4.80(1.51)$ \\
$\begin{array}{l}\text { 2. Cuando quiero sentir una emoción menos negativa, cambio la ma- } \\
\text { nera en que estoy pensando sobre la situación }\end{array}$ & $.85^{* * *}$ & $-.07^{* * *}$ & $5.12(1.47)$ \\
$\begin{array}{l}\text { 3. Cuando quiero sentir una emoción más positiva, cambio la manera } \\
\text { en que estoy pensando sobre la situación }\end{array}$ & $.83^{* * *}$ & $-.07^{* * *}$ & $5.16(1.44)$ \\
$\begin{array}{l}\text { 4. Cuando quiero sentir una emoción más positiva (como alegría o } \\
\text { diversión), cambio lo que estoy pensando }\end{array}$ & $.73^{* * *}$ & .02 & $4.82(1.51)$ \\
$\begin{array}{l}\text { 5. Cuando quiero sentir una emoción menos negativa (como tristeza } \\
\text { o ira), cambio lo que estoy pensando }\end{array}$ & $.72^{* * *}$ & .03 & $4.75(1.50)$ \\
$\begin{array}{l}\text { 6. Cuando me enfrento ante una situación estresante, trato de pensar } \\
\text { sobre ello de forma que me ayude a mantener la calma }\end{array}$ & $.57^{* * *}$ & $-.10^{* * *}$ & $5.48(1.40)$ \\
$\begin{array}{l}\text { Supresión emocional } \\
\text { 7. Controlo mis emociones no expresándolas }\end{array}$ & -.04 & $.72^{\mathrm{a}}$ & $2.04(1.03)$ \\
$\begin{array}{l}\text { 8. Cuando siento emociones negativas, me aseguro de no expresarlas } \\
\text { 9. Guardo mis emociones para mí mismo/a }\end{array}$ & .01 & $.91^{* * *}$ & $3.15(1.85)$ \\
10. Cuando siento emociones positivas, me aseguro de no expresarlas & -.02 & $.89^{* * *}$ & $3.56(1.87)$ \\
a / $\omega$ & -.05 & $.92^{* * *}$ & $3.42(1.99)$ \\
\hline
\end{tabular}

Nota: ${ }^{\mathrm{a}}=$ varianza media extraída (AVE). $\alpha=$ alfa de Cronbach; $\omega=$ omega de McDonald. $* * * p<.001$.

\section{Invarianza}

Para establecer la invarianza temporal del ERQ, en un primer paso, a partir de los resultados de ambas aplicaciones (tiempo 1 y tiempo 2), se calculó el modelo base sin restricciones $\left(\chi_{(66, \mathrm{n}=725)}^{2}=151.30, p<.001\right.$, RMSEA $=$ $.042, \mathrm{SRMR}=.049, \mathrm{CFI}=.990, \mathrm{GFI}=.997, \mathrm{NFI}=.982$, $\mathrm{TLI}=.986$ ). El siguiente paso fue evaluar los cambios en el modelo a partir de la introducción de las restricciones entre las cargas factoriales, los interceptos, los residuos y las medias de los ítems y los factores de ambas aplicacio$\operatorname{nes}\left(\chi_{(94, \mathrm{n}=725)}^{2}=230.32, p<.001, \mathrm{RMSEA}=.045\right.$, SRMR $=.055, \mathrm{CFI}=.983, \mathrm{GFI}=.995, \mathrm{NFI}=.972, \mathrm{TLI}=.984)$. Siguiendo los criterios propuestos por (Cheung y Rensvold, 2002), vamos a considerar diferencias entre los valores en el RMSEA y el CFI menores de -.01, como evidencia de invarianza. Los resultados de esta comparación indican que el ERQ presenta invarianza temporal estricta pasados 6 meses $(\triangle \mathrm{RMSEA}=.003, \Delta \mathrm{CFI}=.007)$.

Siguiendo un proceso similar al establecido con los pasos para calcular la invarianza temporal, se calculó la invarianza entre sexos. Los resultados del modelo base mostraron un adecuado ajuste $\left(\chi_{(66, \mathrm{n}=983)}^{2}=124.61\right.$, $p<.001, \mathrm{RMSEA}=.043, \mathrm{SRMR}=.048, \mathrm{CFI}=.996, \mathrm{GFI}$ $=.999, \mathrm{NFI}=.971, \mathrm{TLI}=.981)$. Las posteriores comparaciones entre los modelos con restricciones mostraron que el ERQ presenta también invarianza estricta entre sexos $(\triangle \mathrm{RMSEA}=.008, \Delta \mathrm{CFI}=.008)$.

\section{Baremos del ERQ}

Finalmente, se calcularon los baremos poblacionales para ambos sexos y grupos de edad (véase la Tabla 6).

\section{Discusión}

El objetivo de la presente investigación ha sido examinar las propiedades psicométricas de la versión española del ERQ. Los resultados de los análisis factoriales sugieren una estructura de dos factores no correlacionados, apoyando así la primera de nuestras hipótesis. Estos resultados son similares a los hallados por los estudios previos sobre validación del cuestionario (Cabello et al., 2013; Gross y John, 2003)

En cuanto al análisis de la validez convergente y discriminante, encontramos que la proporción de va- 
Tabla 5. Estadísticos descriptivos de las escalas, correlaciones entre las dimisiones de regulación emocional y otras variables relevantes, y coeficientes de fiabilidad

\begin{tabular}{|c|c|c|c|c|}
\hline & $M(D T)$ & $\begin{array}{l}\text { Reevaluación } \\
\text { cognitiva }\end{array}$ & Supresión emocional & $\alpha / \omega$ \\
\hline DTS & $3.21(0.83)$ & $.16^{* * *}$ & $-.17 * * *$ & $.90 / .91$ \\
\hline Tolerancia & $3.08(1.14)$ & $.09^{* *}$ & $-.11 * * *$ & $.83 / .85$ \\
\hline Evaluación & $3.57(0.90)$ & $.24 * * *$ & $-.18 * * *$ & $.81 / .80$ \\
\hline Absorción & $3.00(1.15)$ & $.19^{* * *}$ & $-.14 * * *$ & $.86 / .87$ \\
\hline Regulación & $2.83(1.05)$ & $-.08^{*}$ & $-.08^{*}$ & $.79 / .83$ \\
\hline SA-45 & $23.73(20.10)$ & $-.23 * * *$ & $.20 * * *$ & $.95 / .95$ \\
\hline Depresión & $3.94(4.10)$ & $-.16 * * *$ & $.22 * * *$ & $.89 / 90$ \\
\hline Hostilidad & $1.74(2.36)$ & $-.24 * * *$ & $.07 *$ & $.76 / 78$ \\
\hline Sensibilidad Interpersonal & $3.31(3.54)$ & $-.23 * * *$ & $.24 * * *$ & $.85 / .85$ \\
\hline Somatización & $3.17(3.27)$ & $-.07 *$ & .05 & $.81 / .83$ \\
\hline Ansiedad & $3.37(3.18)$ & $-.21 * * *$ & $.10 * * *$ & $.83 / .89$ \\
\hline Psicoticismo & $0.50(1.19)$ & $-.11 * * *$ & $.07^{*}$ & $.60 / .64$ \\
\hline Obsesión-Compulsión & $3.49(3.17)$ & $-.14 * * *$ & $.17 * * *$ & $.78 / .78$ \\
\hline Ansiedad Fóbica (Agorafobia) & $0.90(2.13)$ & $-.20^{* * *}$ & $.15^{* * *}$ & $.84 / .85$ \\
\hline Ideación Paranoide & $3.28(3.19)$ & $-.15^{* * *}$ & $.19 * * *$ & $.78 / .77$ \\
\hline PANAS & - & - & - & - \\
\hline Afecto positivo & $29.86(6.74)$ & $.28 * * *$ & $-.23 * * *$ & $.91 / .92$ \\
\hline Afecto negativo & $18.63(5.69)$ & $-.28 * * *$ & $.16^{* * *}$ & $.89 / .93$ \\
\hline TAS-20 & $41.24(8.65)$ & $-.23 * * *$ & $.44 * * *$ & $.79 / .83$ \\
\hline
\end{tabular}

Nota: $\alpha=$ alfa de Cronbach; $\omega=$ omega de McDonald. * $p<.05 ; * * p<.01 ; * * p<.001$.

rianza media explicada por los factores de ERQ ha sido muy próxima a .50 para la Reevaluación cognitiva (.47) y sobradamente superior en el caso de la Supresión emocional. Siguiendo los criterios establecidos por la literatura previa, hemos de concluir que la escala ha mostrado evidencias de validez convergente (Fornell y Larcker, 1981; Hair, Black, Babin, y Anderson, 2009). Del mismo modo, nuestros resultados también apoyan la hipótesis de validez discriminante. Hasta donde sabemos, es la primera vez que se establece la validez convergente y discriminante de la escala siguiendo criterios estadísticos (Fornell y Larcker, 1981), lo que supone un importante avance en el conocimiento de las propiedades psicométricas de la escala. Hemos constatado datos adicionales sobre validez a través de las correlaciones positivas y estadísticamente significativas entre la supresión emocional y los síntomas psicopatológicos. Esta relación parece especialmente relevante en el caso de la sintomatología relacionada con los trastornos de ansiedad (fobia social, ansiedad fóbica, y ansiedad generalizada), los síntomas depresivos y los sín- tomas del trastorno obsesivo compulsivo. Así mismo, hemos constatado relaciones negativas y significativas entre la reevaluación cognitiva y estos mismos síntomas. En esta línea, diversos estudios han encontrado similar relación con la sintomatología ansioso-depresiva (Aldao et al., 2010; Schafer, Naumann, Holmes, Tuschen-Caffier, y Samson, 2016). Estos hallazgos tienen importantes implicaciones para el conocimiento de la etiología de los trastornos de ansiedad y del estado de ánimo, poniendo de manifiesto el carácter transdiagnóstico de la regulación emocional (Aldao, 2012; Sandín et al., 2012).

Respecto a la fiabilidad de la ERQ, nuestros resultados han mostrado una adecuada fiabilidad de la escala, con valores de alfa de Cronbach superiores a .84, lo que supone una mejora incremental de la fiabilidad comparado con los resultados de Cabello et al. (2013) y Gross y John (2003). Estas diferencias podrían deberse a los ligeros cambios que se aprecian entre nuestra traducción, donde los ítems tal vez podrían resultar más simples y fáciles de interpretar. Futuras investigaciones deberían 
Tabla 6. Descriptivos y baremos poblacionales por sexo y grupos de edad $(N=1.980)$

\begin{tabular}{|c|c|c|c|c|c|c|c|c|c|c|c|c|}
\hline & \multicolumn{6}{|c|}{ Hombres $(n=432)$} & \multicolumn{6}{|c|}{ Mujeres $(n=1.548)$} \\
\hline & \multicolumn{2}{|c|}{$18-25$} & \multicolumn{2}{|c|}{$26-40$} & \multicolumn{2}{|c|}{$>41$} & \multicolumn{2}{|c|}{$18-25$} & \multicolumn{2}{|c|}{$26-40$} & \multicolumn{2}{|c|}{$>41$} \\
\hline & Sup. & Rev. & Sup. & Rev. & Sup. & Rev. & Sup. & Rev. & Sup. & Rev. & Sup. & Rev. \\
\hline$M$ & 16.30 & 29.35 & 15.05 & 29.59 & 14.79 & 29.49 & 12.69 & 29.59 & 12.07 & 30.22 & 12.04 & 30.77 \\
\hline$D T$ & 6.21 & 5.40 & 6.33 & 6.24 & 6.33 & 6.77 & 6.00 & 6.24 & 5.97 & 6.49 & 5.61 & 6.36 \\
\hline Min & 4 & 16 & 4 & 13 & 4 & 11 & 4 & 6 & 4 & 6 & 4 & 6 \\
\hline Max & 28 & 42 & 27 & 42 & 28 & 42 & 28 & 42 & 28 & 42 & 27 & 42 \\
\hline$n$ & 80 & 80 & 229 & 229 & 123 & 123 & 498 & 498 & 726 & 726 & 324 & 324 \\
\hline \multicolumn{13}{|c|}{ Percentiles } \\
\hline 5 & 4 & 18 & 5 & 18 & 8 & 16 & 4 & 17 & 4 & 17 & 4 & 18 \\
\hline 10 & 8 & 23 & 6 & 20 & 5 & 18 & 4 & 22 & 4 & 22 & 5 & 23 \\
\hline 15 & 8 & 23 & 8 & 25 & 7 & 26 & 6 & 24 & 6 & 24 & 6 & 25 \\
\hline 20 & 10 & 25 & 8 & 24 & 8 & 23 & 7 & 26 & 7 & 26 & 7 & 26 \\
\hline 25 & 11 & 26 & 9 & 26 & 9 & 25 & 8 & 27 & 8 & 27 & 8 & 27 \\
\hline 30 & 12 & 27 & 12 & 27 & 11 & 26 & 8 & 28 & 8 & 28 & 8 & 28 \\
\hline 35 & 14 & 28 & 12 & 28 & 12 & 28 & 9 & 29 & 8 & 29 & 9 & 29 \\
\hline 40 & 15 & 29 & 13 & 29 & 13 & 30 & 10 & 30 & 9 & 30 & 9 & 30 \\
\hline 45 & 15 & 29 & 15 & 30 & 14 & 31 & 11 & 30 & 10 & 31 & 10 & 31 \\
\hline 50 & 16 & 29 & 16 & 30 & 16 & 31 & 13 & 31 & 11 & 31 & 11 & 31 \\
\hline 55 & 18 & 30 & 16 & 31 & 17 & 32 & 14 & 31 & 12 & 32 & 12 & 32 \\
\hline 60 & 19 & 31 & 18 & 32 & 18 & 32 & 15 & 32 & 13 & 33 & 13 & 33 \\
\hline 65 & 20 & 32 & 19 & 33 & 19 & 33 & 16 & 33 & 15 & 33 & 15 & 24 \\
\hline 70 & 21 & 33 & 19 & 33 & 19 & 34 & 17 & 34 & 16 & 34 & 16 & 35 \\
\hline 75 & 21 & 33 & 20 & 34 & 20 & 35 & 18 & 34 & 17 & 35 & 17 & 36 \\
\hline 80 & 22 & 34 & 21 & 35 & 20 & 36 & 18 & 35 & 18 & 36 & 18 & 36 \\
\hline 85 & 24 & 34 & 22 & 36 & 21 & 36 & 20 & 36 & 19 & 36 & 19 & 36 \\
\hline 90 & 24 & 36 & 24 & 37 & 24 & 36 & 21 & 36 & 20 & 37 & 20 & 37 \\
\hline 95 & 26 & 39 & 24 & 38 & 24 & 38 & 23 & 38 & 22 & 39 & 21 & 40 \\
\hline 99 & 28 & 42 & 27 & 42 & 27 & 42 & 25 & 42 & 25 & 42 & 25 & 42 \\
\hline
\end{tabular}

Nota Sup. = supresión emocional; Rev. = reevaluación cognitiva.

establecer si estas mejoras se mantienen en otras muestras distintas.

Los resultados del análisis de la estabilidad temporal a 6 meses mediante la técnica de la invarianza factorial han resultado adecuados, lo que nos permite afirmar que se ha cumplido la quinta de nuestras hipótesis, estableciendo así la invarianza temporal de la prueba. Es decir, que los resultados de la escala miden el mismo constructo a lo largo del tiempo, por lo que diversas aplicaciones del test pueden ser comparables en términos de diferen- cias en las dimensiones de la ERQ. Aparte de la importancia que supone probar la hipótesis de la invarianza temporal (Cheung y Rensvold, 2002; Pineda et al., 2018; Widaman et al., 2010), que nosotros sepamos es la primera vez que se establece para cualquiera de las versiones disponibles de esta prueba, lo que de nuevo supone un importante hallazgo.

En lo relativo a la sexta de nuestras hipótesis, los resultados sugieren que la prueba es invariante respecto del sexo de los participantes, mostrando evidencias de 
invarianza estricta. Estos resultados van en línea de lo reportado por Melka y colaboradores (2011). Otras validaciones de la ERQ también han mostrado evidencias de invarianza, lo que confirmaría que la escala estaría midiendo el mismo constructo en diferentes muestras (Liu, Chen, y Tu, 2015). De hecho, el estudio realizado por Sala et al. (2012), en el que se comparan dos versiones diferentes (la alemana y la italiana) vendría a confirmar también la invarianza de la ERQ.

El trabajo presenta algunas limitaciones que debían tenerse en consideración. Las diferencias en el número de mujeres y hombres de la muestra hacen que los resultados deban ser interpretados con cautela. Así mismo, se trata de una muestra de universitarios, y aunque la muestra es heterogénea en los principales factores sociodemográficos (edad, nivel económico, trabajo, familia, distribución geográfica, etc.), puede que los resultados no sean del todo extrapolables a la población española con niveles culturales bajos.

Con todo, el presente trabajo supone una importante aportación para la psicología clínica, al proporcionar nueva evidencia sobre un cuestionario de gran interés y aplicabilidad en este campo. Futuras investigaciones deberían examinar en profundidad la regulación emocional como un constructo transdiagnóstico, y establecer la naturaleza de las relaciones de este constructo con los distintos trastornos de ansiedad y del estado del ánimo, así como también de otras posibles variables transdiagnósticas relevantes, tales como la sensibilidad a la ansiedad, la tolerancia a la incertidumbre o el afecto positivo y negativo. Del mismo modo, también deberá investigarse la relevancia de la inclusión de esta variables en los nuevos protocolos de terapia cognitivo-conductual transdiagnóstica (García-Escalera, Chorot, Valiente, Reales, y Sandín, 2016).

\section{Conflictos de intereses}

Los autores declaran que no existen conflictos de intereses.

\section{Referencias}

Aldao, A. (2012). Emotion regulation strategies as transdiagnostic processes: A closer look at the invariance of their form and function. Revista de Psicopatologia y Psicologia Clinica, 17(3), 261-277.https://doi.org/10.5944/rppc.vol.17.num.3.2012.11843

Aldao, A., y Nolen-Hoeksema, S. (2010). Specificity of cognitive emotion regulation strategies: A transdiagnostic examination. Behaviour Research and Therapy, 48(10), 974-983. https:// doi.org/10.1016/j.brat.2010.06.002

Aldao, A., Nolen-hoeksema, S., y Schweizer, S. (2010). Emotionregulation strategies across psychopathology : A meta-analytic review. Clinical Psychology Review, 30, 217-237. https://doi. org/10.1016/j.cpr.2009.11.004

Amstadter, A. B. (2008). Emotion regulation and anxiety disorders. Journal of Anxiety Disorders, 22(2), 211-221. https://doi.org/10.1016/j.janxdis.2007.02.004.Emotion

Bagby, R. M., Parker, J. D., y Taylor, G. J. (1994). The twenty-item Toronto Alexithymia Scale-I. Item selection and cross-validation of the factor structure. Journal of Psychosomatic Research, 38(1), 23-32. https://doi.org/10.1016/0022-3999(94)90005-1

Cabello, R., Salguero, J. M., Fernández-Berrocal, P., y Gross, J. J. (2013). A Spanish adaptation of the Emotion Regulation Questionnaire. European Journal of Psychological Assessment, 29(4), 234-240. https://doi.org/10.1027/10155759/a000150

Cheung, G. W., y Rensvold, R. B. (2002). Evaluating Goodnessof-Fit Indexes for testing measurement invariance. Structural Equation Modeling: A Multidisciplinary Journal, 9(2), 233255. https://doi.org/10.1207/S15328007SEM0902_5

Cummings, C. M., Caporino, N. E., y Kendall, P. C. (2014). Comorbidity of anxiety and depression in children and adolescents: 20 years after. Psychological Bulletin, 140(3), 816-845. https://doi.org/10.1037/a0034733

Davison, M. L., Bershadsky, B., Bieber, J., Silversmith, D., Maruish, M. E., y Kane, R. L. (1997). Development of a brief, multidimensional, self-report instrument for treatment outcomes assessment in psychiatric settings: Preliminary findings. Assessment, 4(3), 259276. https://doi.org/10.1177/107319119700400306

Dimitrov, D. M. (2010). Testing for Factorial Invariance in the Context of Construct Validation. Measurement and Evaluation in Counseling and Development, 43(2), 121-149. https://doi. org/10.1177/0748175610373459

Dimitrov, D. M. (2012). Statistical Methods for Validation of Assessment Scale Data in Counseling and Related Fields. Alexansria, VA: John Wiley \& Sons.

Dunn, T. J., Baguley, T., y Brunsden, V. (2014). From alpha to omega: A practical solution to the pervasive problem of internal consistency estimation. British Journal of Psychology, 105(3), 399-412. https://doi.org/10.1111/bjop.12046

Fornell, C., y Larcker, D. F. (1981). Evaluating Structural Equation Models with Unobservable Variables and Measurement Error. Journal of Marketing Research, 18(1), 39. https://doi. org/10.2307/3151312

García-Escalera, J., Chorot, P., Valiente, R. M., Reales, J. M., y Sandín, B. (2016). Efficacy of transdiagnostic cognitivebehavioral therapy for anxiety and depression in adults, children and adolescents: A meta-analysis. Revista de Psicopatología y Psicología Clínica, 21(3), 147-175.

https://doi.org/10.5944/rppc.vol.21.num.3.2016.17811

García-Escalera, J., Valiente, R. M., Chorot, P., Ehrenreich-May, J., Kennedy, S. M., \& Sandín, B. (2017). The Spanish version of the Unified Protocol for Transdiagnostic Treatment of Emotional Disorders in Adolescents (UP-A) adapted as a school-based anxiety and depression prevention program: Study protocol of a cluster randomized controlled trial. JMIR Research Protocols, doi:10.2196/resprot.7934.

Gil-Sanz, D., Fernández-Modamio, M., Bengochea-Seco, R., Arrieta-Rodríguez, M., González-Fraile, E., Pérez-Fuentes, G., ... y Santos-Zorrozúa, B. (2017). PERE: Una nueva herramienta para valorar el reconocimiento de las emociones 
básicas y su aplicación en la esquizofrenia. Revista de Psicopatología y Psicología Clínica, 22(2), 85-93.

Grill, S. S., Castañeiras, C., y Fasciglione, M. P. (2017). Aplicación grupal del Protocolo Unificado para el tratamiento transdiagnóstico de los trastornos emocionales en población argentina. Revista de Psicopatología y Psicología Clínica, 22(3), 171-181. https://doi.org/10.5944/rppc.vol.22. num.3.2017.18122

Gross, J. J. (2002). Emotion regulation : Affective, cognitive, and social consequences. Psychophysiology, 39, 281-291.

Gross, J. J., y John, O. P. (2003). Individual differences in two emotion regulation processes: implications for affect, relationships, and well-being. Journal of Personality and Social Psychology, 85(2), 348-362. https://doi.org/10.1037/0022-3514.85.2.348

Hair, J. F., Black, W. C., Babin, B. J., y Anderson, R. E. (2010). Multivariate Data Analysis. New Jersey: Pearson.

Hair, J. F., Sarstedt, M., Hopkins, L., y Kuppelwieser, V. G. (2014). Partial least squares structural equation modeling (PLSSEM). European Business Review, 26(2), 106-121. https:// doi.org/10.1108/EBR-10-2013-0128

Jiménez Ros, A. M., Orgambidez Ramos, A., Pascual Orts, L. M.. (2015). Inventario de supresión del pensamiento del «oso blanco»(WBSI): Propiedades psicométricas de la versión portuguesa (Portugal). Revista de Psicopatología y Psicología Clínica, 20(2), 125-134.

Korkmaz, S., Goksuluk, D., y Zararsiz, G. (2014). MVN: An R package for assessing multivariate normality. The R Journal, 6(2013), 151-162.

Liu, W., Chen, L., y Tu, X. (2015). Chinese adaptation of Emotion Regulation Questionnaire for Children and Adolescents (ERQ-CCA): A psychometric evaluation in Chinese children. International Journal of Psychology, 52(5), 398-405. https:// doi.org/10.1002/ijop.12233

Lopes, P. N., Salovey, P., Côté, S., y Beers, M. (2005). Emotion Regulation Abilities and the Quality of Social Interaction. Emorion, 5(1), 113-118. https://doi.org/10.1037/1528-3542.5.1.113

Martin, R. C., Dahlen, E. R., Martin, R. C., y Dahlen, E. R. (2005). Cognitive emotion regulation in the prediction of depression, anxiety, stress, and anger depression, anxiety, stress, and anger. Personality and Individual Difference, 39, 1249-1260. https://doi.org/10.1016/j.paid.2005.06.004

Martínez-Vispo, C., Fernández del Río, E., López-Durán, A., y Becoña, E. (2016). Influencia de la sensibilidad a la ansiedad en una intervención psicológica para dejar de fumar. Revista de Psicopatología y Psicología Clínica, 21(1), 11-19.

Mayer, J. D., Caruso, D. R., y Salovey, P. (1999). Emotional Intelligence Meets Traditional Standards for an Intelligence. Intelligence, 27(4), 267-298. https://doi.org/10.1016/S01602896(99)00016-1

McLaughlin, K. A., y Nolen-Hoeksema, S. (2011). Rumination as a transdianostic factor in depression and anxiety. Behaviour Research and Therapy, 49(3), 186-193. https://doi. org/10.1016/j.brat.2010.12.006.Rumination

Meade, A. W., Johnson, E. C., y Braddy, P. W. (2006). The utility of alternative Fit Indices in tests of measurement invariance. Academy of Management Proceedings, 2006(1), B1-B6. https://doi.org/10.5465/AMBPP.2006.27182124

Meade, A. W., Johnson, E. C., y Braddy, P. W. (2008). Power and Sensitivity of Alternative Fit Indices in tests of measurement invariance. Journal of Applied Psychology, 93(3), 568-592. https://doi.org/10.5465/AMBPP.2006.27182124

Meade, A. W., y Lautenschlager, G. J. (2004). A monte-carlo study of confirmatory factor analytic tests of measurement equivalence/invariance. Structural Equation Modeling, 11(1), 60-72. https://doi.org/10.1207/S15328007SEM1101_5

Melka, S. E., Lancaster, S. L., Bryant, A. R., y Rodriguez, B. F. (2011). Confirmatory factor and measurement invariance analyses of the emotion regulation questionnaire. Journal of Clinical Psychology, 67(12), 1283-1293. https://doi.org/10.1002/jclp.20836

Meredith, W. (1993). Measurement invariance, factor analysis and factorial invariance. Psychometrika, 58(4), 525-543. https:// doi.org/10.1007/BF02294825

Pineda, D., Martín-Vivar, M., Sandín, B., y Piqueras, J. A. (2018). Factorial invariance and norms of the 30-item shortenedversion of the Revised Child Anxiety and Depression Scale (RCADS-30). Psicothema, 30(2), 1-6. https://doi.org/10.7334/ psicothema2017.276

Piqueras, J. A., Martín-Vivar, M., Sandín, B., San Luis, C., y Pineda, D. (2017). The Revised Child Anxiety and Depression Scale: A systematic review and reliability generalization meta-analysis. Journal of Affective Disorders, 218(15), 153169. https://doi.org/10.1016/j.jad.2017.04.022

$\mathrm{R}$ Core Team. (2016). R: A language and environment for statistical computing. R Foundation for Statistical Computing. Vienna, Austria.

Rhemtulla, M., Brosseau-Liard, P. É., y Savalei, V. (2012). When can categorical variables be treated as continuous? A comparison of robust continuous and categorical SEM estimation methods under suboptimal conditions. Psychological Methods, 17(3), 354-373. https://doi.org/10.1037/a0029315

Rodríguez-Carvajal, R., Moreno-Jiménez, B., y Garrosa, E. (2006). Emotion Regulation Questionnaire - Spanish version. Recuperado a partir de https://spl.stanford.edu/resources

Rosique, M. T., Sandín, B., Valiente, R. M., García-Escalera, y Chorot, P. (en prensa). Tratamiento transdiagnóstico de un caso clínico con elevada comorbilidad de trastornos emocionales: Aplicación del Protocolo Unificado. Revista Argentina de Clínica Psicológica

Rosseel, Y. (2012). lavaan: An R package for structural equation mdeling. Journal of Statistical Software, 48(2), 1-36.

Sala, M. N., Molina, P., Abler, B., Kessler, H., Vanbrabant, L., y van de Schoot, R. (2012). Measurement invariance of the Emotion Regulation Questionnaire (ERQ). A cross-national validity study. European Journal of Developmental Psychology, 9(6), 751-757. https://doi.org/10.1080/17405629 .2012 .690604

Salovey, P., y Mayer, J. (1990). Emotional Intelligence. Imagination Cognition and Personality, 9, 185-211. https://doi. org/10.2190/DUGG-P24E-52WK-6CDG

Sánchez-Arribas, C., Chorot, P., Valiente, R. M., y Sandín, B. (2015). Evaluación de factores cognitivos positivos y negativos relacionadas con el trastorno de pánico: Validación del CATP. Revista de Psicopatología y Psicología Clínica, 20(2), 85-100.

Sandín, B., Chorot, P., Lostao, L., Joiner, T. E., Santed, M. A., y Valiente, R. M. (1999). Escalas PANAS de afecto positivo y negativo: Validación factorial y convergencia transcultural. Psicothema, 11(1), 37-51. 
Sandín, B., Chorot, P., Santed, M. A., y Jiménez, P. (1996). Dimensiones de alexitimia y estados emocionales de ansiedad, depresión e ira. Psiquis: Revista de Psiquiatría, Psicología Médica y Psicosomática, 17(1), 49-57.

Sandín, B., Chorot, P., y Valiente, R. M. (2012). Transdiagnóstico: Nueva frontera en psicología clínica. Revista de Psicopatologia y Psicologia Clinica, 17(3), 185-203. https://doi.org/10.5944/ rppc.17.3.11839

Sandín, B., Chorot, P., y Valiente, R. M., y Pineda, D. (2012). Versión española del cuestionario ERQ. Madrid: UNED.

Sandín, B., Simons, J. S., Valiente, R. M., Simons, R. M., y Chorot, P. (2017). Psychometric properties of the spanish version of The Distress Tolerance Scale and its relationship with personality and psychopathological symptoms. Psicothema, 29(3), 421-428. https://doi.org/10.7334/psicothema2016.239

Sandín, B., Valiente, R. M., Chorot, P., Santed, M. A., y Lostao, L. (2008). SA-45: Forma abreviada del SCL-90. Psicothema, 20(2), 290-296.
Schafer, J. O., Naumann, E., Holmes, E. A., Tuschen-Caffier, B., y Samson, A. C. (2016). Emotion regulation strategies in depressive and anxiety symptoms in youth: A meta-analytic review. Journal of Youth and Adolescence, 1-16. https://doi. org/10.1007/s10964-016-0585-0

Segura, M., Roncero, M., Oltra-Cucarella, J., Blasco, L., Ciscar, S., Portillo, M., ... y Perpiñá, C. (2017). Entrenamiento en remediación cognitiva y habilidades emocionales en formato grupal para pacientes con obesidad: Un estudio piloto. Revista de Psicopatología y Psicología Clínica, 22(2), 127-138.

Simons, J. S., y Gaher, R. M. (2005). The distress tolerance scale: Development and validation of a self-report measure. Motivation and Emotion, 29(2), 83-102. https://doi. org/10.1007/s11031-005-7955-3

Widaman, K. F., Ferrer, E., y Conger, R. D. (2010). Factorial invariance within longitudinal structural equation models: Measuring the same construct across time. Child Development Perspectives, 4(1), 10-18. https://doi.org/10.1111/j.1750-8606.2009.00110.x 